\title{
Carboxyethylpyrrole oxidative protein modifications stimulate neovascularization: Implications for age-related macular degeneration
}

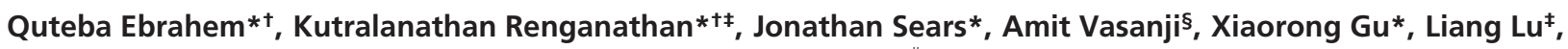 \\ Robert G. Salomon ${ }^{\ddagger}$, John W. Crabb*ะ§ף, and Bela Anand-Apte* ${ }^{* \S \|}$ \\ *Cole Eye Institute and §Lerner Research Institute, Cleveland Clinic Foundation, and `Department of Chemistry, Case Western Reserve University, \\ Cleveland, $\mathrm{OH} 44195$
}

Edited by Mark T. Keating, Novartis Institutes for Biomedical Research, Cambridge, MA, and approved July 17, 2006 (received for review February 27, 2006)

Choroidal neovascularization (CNV), the advanced stage of agerelated macular degeneration (AMD), accounts for $>80 \%$ of vision loss in AMD. Carboxyethylpyrrole (CEP) protein modifications, uniquely generated from oxidation of docosahexaenoate-containing lipids, are more abundant in Bruch's membrane from AMD eyes. We tested the hypothesis that CEP protein adducts stimulate angiogenesis and possibly contribute to CNV in AMD. Human serum albumin (HSA) or acetyl-Gly-Lys-O-methyl ester (dipeptide) were chemically modified to yield CEP-modified HSA (CEP-HSA) or CEP-dipeptide. The in vivo angiogenic properties of CEP-HSA and CEP-dipeptide were demonstrated by using the chick chorioallantoic membrane and rat corneal micropocket assays. Low picomole amounts of CEP-HSA and CEP-dipeptide stimulated neovascularization. Monoclonal anti-CEP antibody neutralized limbal vessel growth stimulated by CEP-HSA, whereas anti-VEGF antibody was found to only partially neutralize vessel growth. Subretinal injections of CEP-modified mouse serum albumin exacerbated laser-induced CNV in mice. In vitro treatments of human retinal pigment epithelial cells with CEP-dipeptide or CEP-HSA did not induce increased VEGF secretion. Overall, these results suggest that CEP-induced angiogenesis utilizes VEGF-independent pathways and that anti-CEP therapeutic modalities might be of value in limiting CNV in AMD.

oxidation

$\mathbf{T}$ he clinical significance of ocular angiogenesis is enormous, largely because of choroidal neovascularization $(\mathrm{CNV})$ in age-related macular degeneration (AMD) (1-3), which is the chief cause of irreversible loss of vision in elderly patients in the western hemisphere. CNV involves abnormal vessel growth from the choriocapillaris through Bruch's membrane resulting in hemorrhage, scarring, exudation, and/or retinal detachment, with the ultimate consequence of a severe loss of high-acuity central vision. The molecular mechanisms involved in the development of $\mathrm{CNV}$ are not well defined, but the need for effective therapies to prevent and treat $\mathrm{CNV}$ is augmented with an increase in the population of people over the age of 65 years.

AMD is a progressive, multifactorial, polygenic disease with poorly understood etiology. Early stages of the disease are typically termed "dry" AMD and are associated with the macular accumulation of extracellular deposits (drusen) below the retinal pigment epithelium (RPE) on Bruch's membrane. Geographic atrophy develops in the later stages of dry AMD and is characterized by macular loss of RPE and photoreceptor cells. Advanced-stage disease or "wet" AMD is characterized by CNV. Oxidative damage has long been suspected of contributing to $\operatorname{AMD}(4,5)$, supported by indirect evidence that smoking significantly increases the risk of AMD (6) and that antioxidant vitamins and zinc can slow the progression of the disease for select individuals (7). Several laboratories have recently shown an association between variants in the complement factor $\mathrm{H}$ gene and susceptibility to AMD (8-12), implicating inflammatory processes in the pathophysiology of the disease $(13,14)$. In addition, the recent observation that aged mice exhibiting the apolipoprotein E4 genotype develop a full range of AMD-like pathologies, including CNV, when fed a high-cholesterol diet suggests that lipid oxidation in combination with genetic and environmental factors might contribute to AMD (15). Our previous proteomic study of drusen established a molecular link between oxidative damage and AMD (16) and demonstrated elevated oxidative protein modifications in AMD tissues. Specifically, 2- $(\omega-$ carboxyethyl)pyrrole (CEP), a unique protein modification derived from the oxidation of docosahexaenoate (DHA)-containing lipids, was found to be more abundant in AMD compared with normal ocular tissues (16) and was localized in Bruch's membrane between the blood-bearing choriocapillaris and RPE. The outer segments of the photoreceptors contain high concentrations of polyunsaturated fatty acids (PUFAs), especially DHA, in the membranes and are exposed to relatively high oxygen tension, close to that found in arterial blood. The photooxidative environment in the retina and the DHA-rich photoreceptor outer segments provide a ready source of reactive oxygen species for generating oxidative modifications. Given the well known susceptibility of PUFAs to undergo oxidation in the presence of oxygen or oxygen-derived radical species, as well as our additional observation of elevated levels of CEP adducts and CEP autoantibodies in AMD plasma (17), we hypothesized that these oxidative protein modifications may be a primary catalyst in drusen formation and may possibly play a role in the development of CNV in AMD (16). Here we provide compelling evidence that CEP protein modifications induce angiogenesis in vivo and augment laser-induced $\mathrm{CNV}$ in a mouse model.

\section{Results}

CEP-Induced Angiogenesis in Chicken Embryo. The potential consequence of CEP-modified human serum albumin (CEP-HSA) on angiogenesis was examined by using the chick chorioallantoic membrane (CAM) assay. The angiogenic response to methylcellulose discs containing $0.17 \mu \mathrm{g}(n=4), 0.3 \mu \mathrm{g}(n=3)$, and $3.3 \mu \mathrm{g}$ $(n=4)$ of CEP-HSA or unmodified human serum albumin (HSA) $(n=8)$ was analyzed. The protein preparations were analyzed for

Conflict of interest statement: J.W.C. is a consultant for Alcon Research Ltd. and obtains research funding from Merck \& Co. and Johnson \& Johnson. J.W.C. and R.G.S. each have license for CEP as an inventor from Franz Biomarkers LLC.

This paper was submitted directly (Track II) to the PNAS office.

Abbreviations: AMD, age-related macular degeneration; CEP, 2-( $\omega$-carboxyethyl)pyrrole; CEP-dipeptide, CEP-modified acetyl-Gly-Lys-O-methyl ester; HSA, human serum albumin CEP-HSA, CEP-modified HSA; MSA, mouse serum albumin; CEP-MSA, CEP-modified MSA; CNV, choroidal neovascularization; CAM, chick chorioallantoic membrane; DHA, docosahexaenoate; PUFA, polyunsaturated fatty acid; RPE, retinal pigment epithelium.

${ }^{\dagger} \mathrm{Q}$.E. and K.R. contributed equally to this work.

ITo whom correspondence may be addressed at: Cole Eye Institute (i31), Lerner Research Institute, Cleveland Clinic Foundation, 9500 Euclid Avenue, Cleveland, OH 44195. E-mail: crabbj@ccf.org.

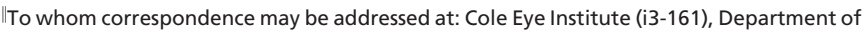
Ophthalmology, Cleveland Clinic Lerner College of Medicine at Case Western Reserve University, Cleveland Clinic Foundation, 9500 Euclid Avenue, Cleveland, OH 44195. E-mail: anandab@ccf.org.

C 2006 by The National Academy of Sciences of the USA 

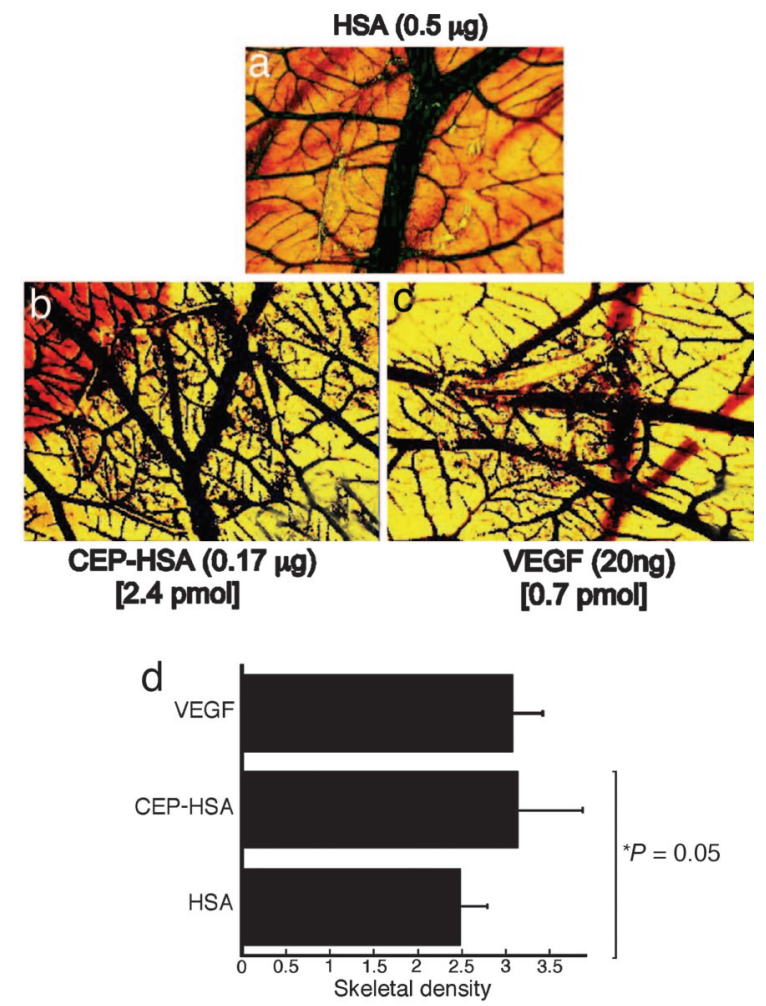

Fig. 1. DHA lipid-derived oxidatively modified HSA (CEP-HSA) induces angiogenesis in the CAM assay. (a-c) CAMs were exposed to pellets containing HSA $(0.5 \mu \mathrm{g}, \approx 7.5 \mathrm{pmol})(a)$, CEP-HSA $(0.17 \mu \mathrm{g}, \approx 2.4 \mathrm{pmol})(b)$, or VEGF ( $20 \mathrm{ng}$, $0.7 \mathrm{pmol}$ ) (c) on day 6 . Two days later the vessels were injected with india ink and visualized by using a stereomicroscope (Olympus, Melville, NY). (d) Skeletal density was quantitated as described in Methods.

endotoxin and were determined to be free of contamination (data not shown). Representative results from these CAM assays are depicted in Fig. 1. CEP-HSA (Fig. $1 b$ ) induced sprouting of new blood vessels that appeared to be tortuous and leaky when perfused with india ink. The average skeletal density of CAM vessels with $0.17 \mu \mathrm{g}$ of CEP-HSA was $\approx 3.1$. Unmodified HSA $(0.5 \mu \mathrm{g})$ did not show this effect (Fig. 1a), with quantitation revealing a lower background skeletal density of $\approx 2.5$. VEGF (20 ng) was used as a positive control (Fig. 1c) and showed an average skeletal density of $\approx 3.1$ in the CAM assay. CEP-HSA $(0.17 \mu \mathrm{g})$ induced a maximal response compared with minimal or absent response with HSA at doses up to $0.5 \mu \mathrm{g}$. The angiogenic response of $0.17 \mu \mathrm{g}$ of CEP-HSA (2.4 pmol) was similar to the half-maximal response of VEGF at a dose of $20 \mathrm{ng}(0.7 \mathrm{pmol})$.

CEP-Induced Angiogenesis in Rat Cornea. The results from the CAM assay were confirmed and extended in rats by using an additional in vivo angiogenesis assay, the corneal micropocket assay. Pellets containing CEP-HSA $(1 \mu \mathrm{g})$ (Fig. $2 d)$ or CEP-modified acetylGly-Lys-O-methyl ester (CEP-dipeptide) (37 ng) (Fig. 2f) when implanted $1 \mathrm{~mm}$ from the limbus of rat cornea stimulated the growth of limbal blood vessels toward the pellet. The newly formed capillaries reached the pellet by day 7 in all of the animals implanted with $1 \mu \mathrm{g}$ or more of CEP-HSA $(n=5)$ or $37 \mathrm{ng}$ or more of CEP-dipeptide $(n=7)$. Notably, unmodified HSA $(1 \mu \mathrm{g}$; $n=3$ ) (Fig. $2 c$ ) or dipeptide (41 ng; $n=6$ ) (Fig. $2 e$ ) did not induce this effect. Pellets containing no protein or peptide were used as a negative control (Fig. $2 a$ ), and VEGF pellets (100 ng) (Fig. 2b) generated the positive control. A statistically significant increase in peak vessel extensions was observed in response to
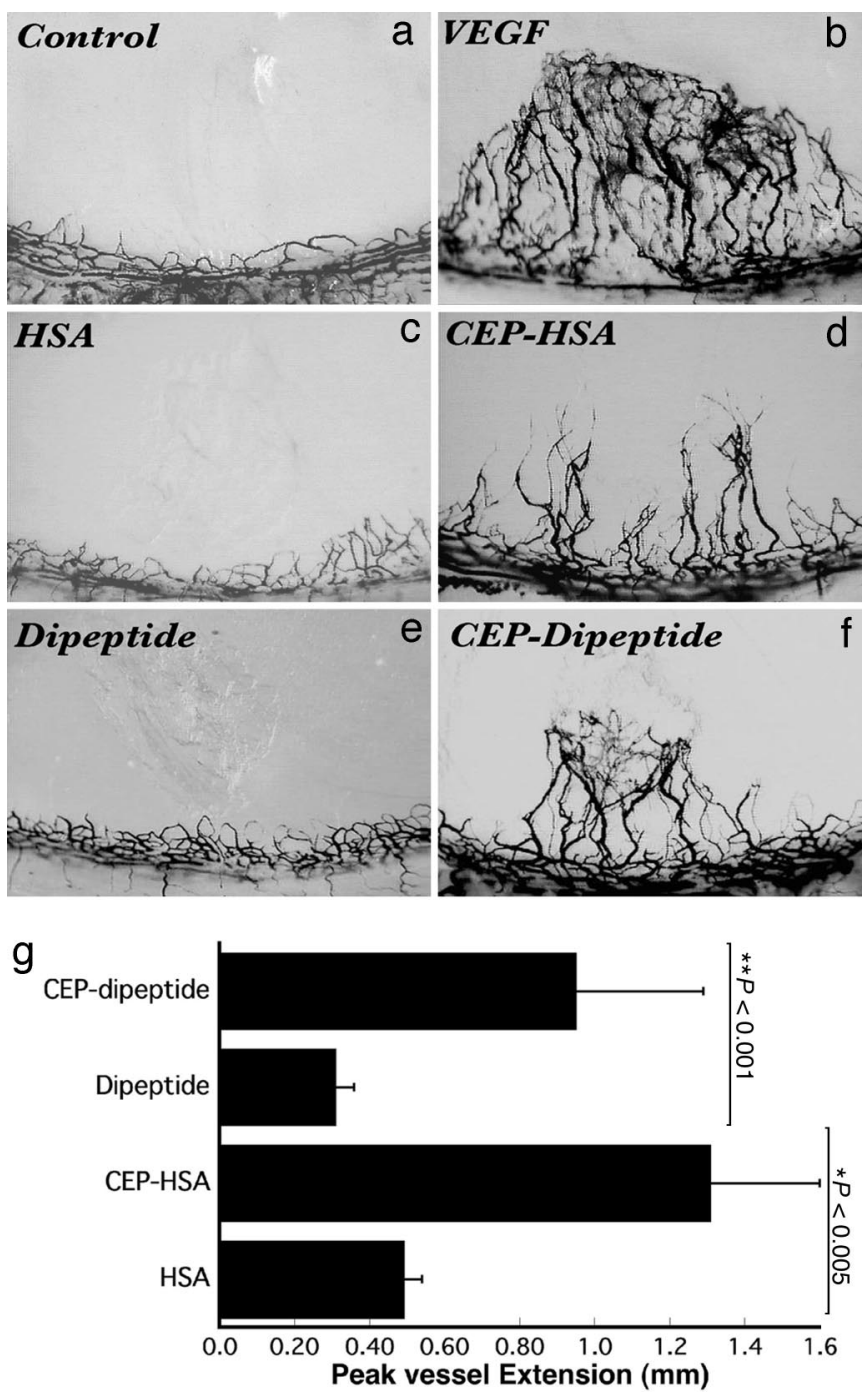

Fig. 2. CEP-HSA as well as dipeptide (Ac-Gly-Lys-OH) induces angiogenesis in a rat corneal micropocket assay. $(a-f)$ Shown are representative photographs of mouse corneas at 7 days after implantation of pellets containing PBS (control) (a), VEGF (100 ng, $\approx 3.5 \mathrm{pmol})(b), \mathrm{HSA}(1 \mu \mathrm{g}, \approx 15 \mathrm{pmol})(c)$, CEP-HSA $(1 \mu \mathrm{g}, \approx 15 \mathrm{pmol})(\mathrm{d})$, dipeptide $(41 \mathrm{ng}, \approx 112 \mathrm{pmol})$ (e), or CEP-dipeptide (37 $\mathrm{ng}, 101 \mathrm{pmol})(f)$. $(g)$ Peak vessel extension was quantitated as described in Methods.

CEP-HSA $(\approx 2.7$ fold) or CEP-dipeptide $(\approx 3.1$ fold $)$ when compared with unmodified parent molecules (Fig. $2 g$ ).

Neutralization of CEP-Induced Angiogenesis with Anti-CEP but Not Anti-VEGF Antibodies. To confirm that the angiogenesis was induced by CEP modification of HSA, pellets were prepared by premixing anti-CEP antibody or anti-VEGF antibody and CEP-HSA. The monoclonal anti-CEP antibody almost completely inhibited the formation of new blood vessels from CEP-HSA implants (Fig. 3d) in the corneal micropocket assay. Neutralizing VEGF antibody only partially inhibited the CEP-HSA-induced neovascularization response (Fig. $4 a, b$, and $e$ ) while completely inhibiting VEGFinduced response (Fig. $4 c$ and $d$ ). Control mouse IgM or IgG antibodies did not show inhibition of CEP-HSA-mediated corneal neovascularization (Figs. $3 c$ and $4 e$ ). Quantitation of peak vessel extensions indicates that the observed neutralization of CEP-HSAinduced angiogenesis by anti-CEP was of greater statistical significance than by anti-VEGF antibodies (Figs. $3 e$ and $4 e$ ). 

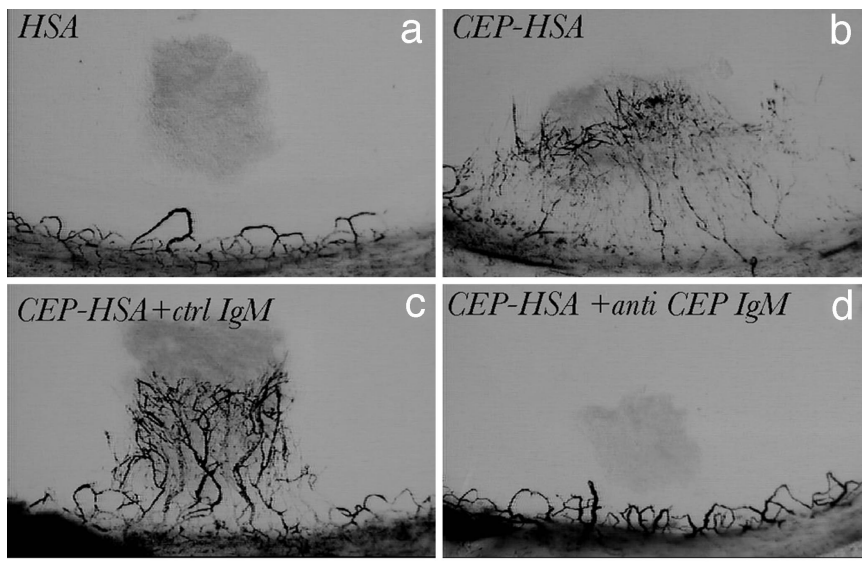

e

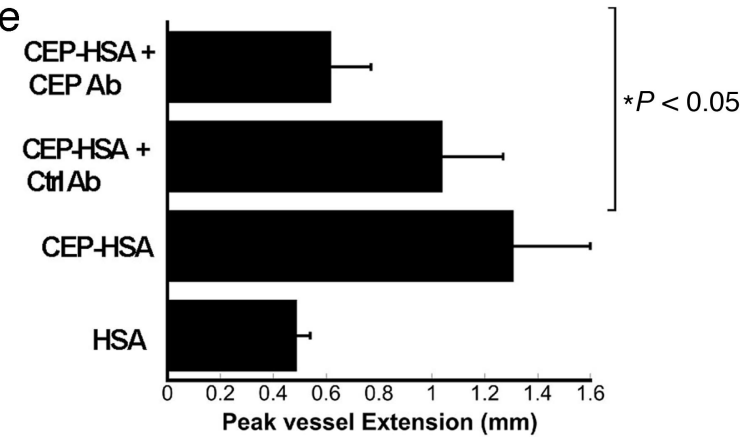

Fig. 3. Neutralization of CEP-HSA-induced angiogenesis by monoclonal antiCEP antibody. $(a-d)$ Shown are representative photographs of mouse corneas at 7 days after implantation of pellets containing HSA ( $1 \mu \mathrm{g}, \approx 149 \mathrm{pmol})(\mathrm{a})$, CEP-HSA $(1 \mu \mathrm{g}, \approx 15 \mathrm{pmol})(b)$, CEP-HSA $(1 \mu \mathrm{g})$ with nonspecific mouse lgM control antibody (c), and CEP-HSA $(1 \mu \mathrm{g})$ with monoclonal anti-CEP antibody (d). (e) Peak vessel extension was quantitated as described in Methods.

CEP-Induced Exacerbation of CNV in a Mouse Model. To determine whether the increased levels of CEP-HSA in AMD Bruch's membrane might contribute to the development of $\mathrm{CNV}$ we examined the effect of CEP-modified mouse serum albumin (CEP-MSA) in a mouse model of CNV. Mice with laser-induced rupture of Bruch's membrane were given a subretinal injection of PBS, mouse serum albumin (MSA), CEP-MSA, or VEGF and analyzed for the development of choroidal neovascular membranes. Quantitative image analysis determined that subretinal injection of CEP-MSA (Fig. $5 c$ and $e$ ) significantly augmented the CNV area when compared with injections of PBS (Fig. $5 a$ and $e$ ) or unmodified MSA (Fig. $5 b$ and $e$ ) and was similar to that obtained with injections of VEGF (Fig. $5 d$ and $e$ ). Subretinal injections of CEP-MSA, MSA, or VEGF in the absence of laser injury did not induce any CNV (data not shown).

CEP Adducts Do Not Stimulate VEGF Secretion in Vitro. Our initial interest in the angiogenic potential of CEP adducts was raised by reports that another type of oxidized protein modification, namely advanced glycation end products, stimulate angiogenesis in vivo (18) and induce VEGF secretion in vitro $(19,20)$. To explore whether CEP adducts influence VEGF secretion in vitro, human RPE cells were treated with CEP-dipeptide $(0.1-10 \mu \mathrm{M})$ and VEGF protein in the cell culture supernatant quantified by ELISA. CEP-dipeptide-treated primary RPE cells or ARPE19 cells did not exhibit increased VEGF in the growth media relative to the unmodified dipeptide or media alone (Fig. 6, which is published as supporting information on the PNAS web site). ARPE19 cells treated with CEP-HSA $(0.1-10 \mu \mathrm{M})$ also exhibited no increase in VEGF secretion (data not shown).
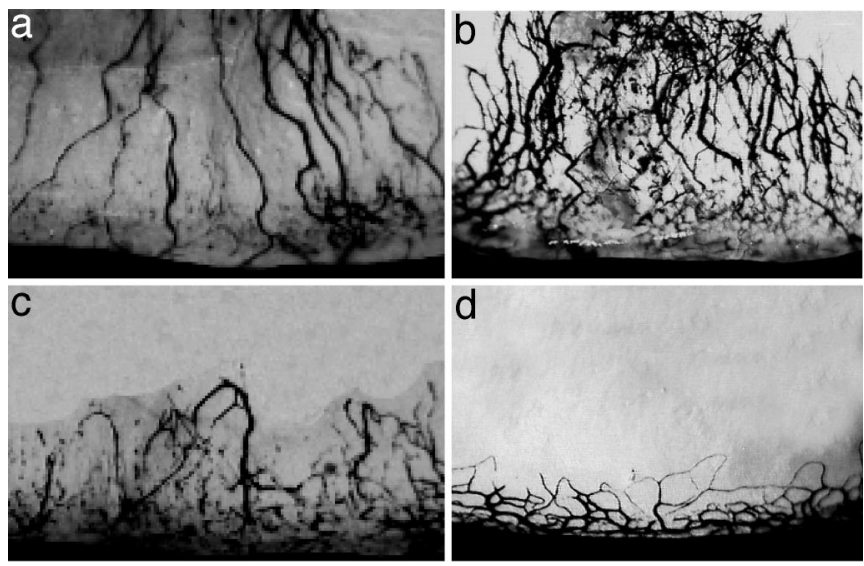

d
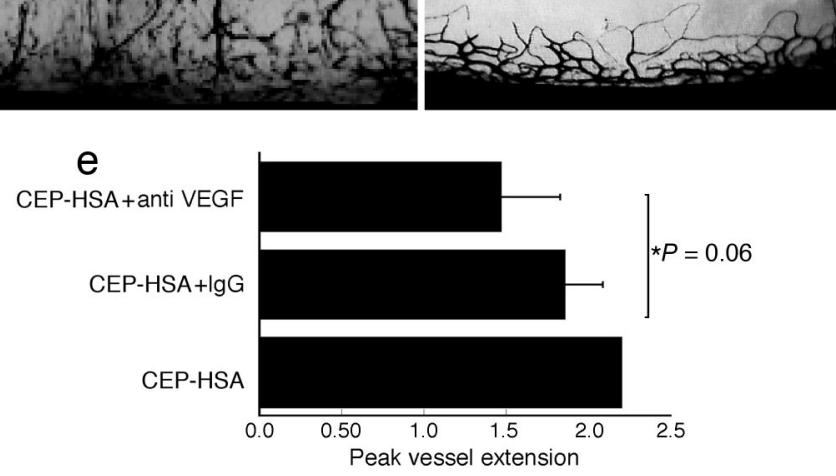

Fig. 4. Incomplete neutralization of CEP-HSA-induced angiogenesis by monoclonal anti-VEGF antibody. ( $a-d)$ Shown are representative photographs of mouse corneas at 7 days after implantation of pellets containing CEP-HSA $(1 \mu \mathrm{g}, \approx 15 \mathrm{pmol})(a)$, CEP-HSA $(1 \mu \mathrm{g})$ with anti-VEGF neutralizing antibody $(b)$, VEGF (20 ng, $\approx 0.7 \mathrm{pmol})(c)$, and VEGF (20 ng) with anti-VEGF neutralizing antibody $(d)$. (e) Peak vessel extension was quantitated as described in Methods.

\section{Discussion}

CEP protein adducts belong to a family of 2-( $\omega$-carboxyalkyl) pyrrole adducts generated from the oxidation of PUFAs (21). For example, oxidative fragmentation of linoleic acid or arachidonic acid can generate 2-( $\omega$-carboxyheptyl)pyrrole or 2-( $\omega$-carboxypropyl)pyrrole adducts, respectively. The phosphatidylcholine esters of the oxidatively truncated PUFA progenitors of these adducts are biologically active and present in atherosclerotic plaques (22-25). However, whereas 2- $(\omega$ carboxypropyl)pyrrole or 2-( $\omega$-carboxyheptyl)pyrrole protein adducts can also arise from oxidation of other common PUFAs, CEP protein adducts uniquely are generated from oxidation of DHA (26). Although rare in most human tissues, DHA accounts for $\approx 80 \mathrm{~mol} \%$ of the polyunsaturated lipids in photoreceptor outer segments (27). The abundance of DHA in photoreceptors and the high photooxidative stress in the retina, as well as the fact that DHA is the most oxidizable fatty acid in humans, all contribute to the higher levels of CEP adducts in AMD. Interestingly, CEP immunoreactivity and CEP autoantibody titer are also significantly elevated in plasma from AMD donors (17) and may be of diagnostic utility as biomarkers for predicting AMD susceptibility. Other oxidative modifications, such as advanced glycation end products, generated from oxidized carbohydrate products also accumulate during aging $(28,29)$, especially in the choriocapillaris, Bruch's membrane (28), and CNV membranes (30). Several studies have shown that advanced glycation end products can stimulate the proliferation of choroid endothelial cells, the expression of matrix metalloproteinase type 2, and growth factors such as VEGF (31) and angiogenesis in vivo (18).

The present study demonstrates that CEP adducts stimulate angiogenesis in the CAM and corneal micropocket assays and 

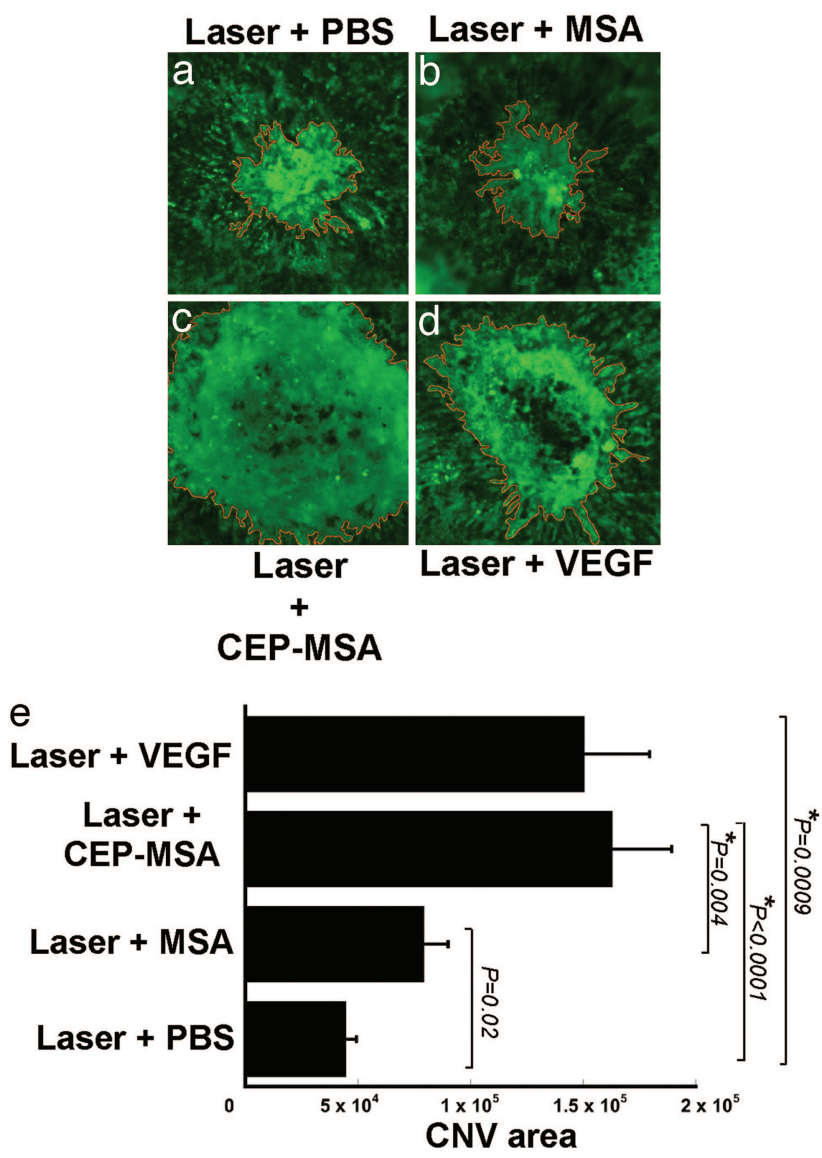

Fig. 5. Exacerbation of CNV lesion by CEP-MSA in a mouse model of CNV. $(a-d)$ Shown are representative photographs of mouse choroids at 14 days after laser photocoagulation and subretinal injections of PBS (a), MSA (1 $\mu \mathrm{g})$ (b), CEP-MSA $(1 \mu \mathrm{g})(c)$, and VEGF $(20 \mathrm{ng})(d)$. (e) CNV area was quantitated as described in Methods.

exacerbate CNV in a mouse model. These results, coupled with the elevated levels of CEP adducts in AMD tissues, strongly suggest that CEP may play a role in the development of the wet (exudative) form of AMD. However, the molecular mechanism by which CEP induces angiogenesis has not yet been determined. An indirect mechanism for in vivo CEP stimulation of angiogenesis might be that CEP induces the release of angiogenic factors such as VEGF or basic FGF by epithelial cells or inhibits the secretion of angiogenesis inhibitors that might contribute to the induction of angiogenesis. The ability of VEGF-neutralizing antibody to only partially block CEP-HSA-induced angiogenesis in vivo and the lack of increase in VEGF secretion in RPE cells exposed to CEP-dipeptide or CEP-HSA suggest the utilization of additional VEGF-independent pathways. Anti-VEGF therapies with recombinant humanized anti-VEGF monoclonal antibody or aptamer are being evaluated as promising treatments for CNV (32-35). Additional studies are needed to determine whether CEP modifications of proteins play a critical role in the development of CNV in AMD and whether CEP neutralization modalities will be effective independently or as a complement to anti-VEGF therapies for the inhibition of CNV in AMD.

\section{Methods}

Synthesis of CEP-Modified Proteins and Peptides. Unambiguous production of CEP was established by using the $\gamma$-ketoaldehyde 4,7-dioxoheptanoic acid as described previously (26). Paal-Knorr condensation of 4,7-dioxoheptanoic acid with HSA and with the dipeptide acetyl-Gly-Lys- $O$-methyl ester was used to generate CEP-
HSA and CEP-dipeptide, respectively, which were characterized by mass spectrometry and NMR as described previously (26). Pyrrole concentration was determined by Ehlrich's assay with 4-(dimethyamino)benzaldehyde and absorbance at $570 \mathrm{~nm}$. Protein was quantified by amino acid analysis (36) and the Bradford protein assay.

CAM Angiogenesis Assay. The CAM assay was performed as described previously (37) with slight modifications. Fertilized 3-dayold white Leghorn eggs (Case Western Reserve University, Squire Valley Farms) were cracked, and embryos with the yolk intact were placed in 100-mm $\times 20-\mathrm{mm}$ glass-bottom Petri dishes. After incubation for 3 days at $37^{\circ} \mathrm{C}$ in $3 \% \mathrm{CO}_{2}$, a methylcellulose disk (Fisher Scientific, Fair Lawn, NJ) containing CEP-HSA or CEPdipeptide was placed on the CAM of individual embryos. CAMs implanted with discs loaded with unmodified dipeptide, HSA, control buffer, or VEGF were used as negative and positive controls, respectively. After $48 \mathrm{~h}$ of incubation, india ink was injected into the vascular system for better visualization of the vessels by a stereomicroscope. Images were captured with a CCD camera (Panasonic, Secaucus, NJ). Samples were always compared on the same CAM to avoid egg-to-egg variability. For quantitative analysis of vessel density and leakage, CAM images were batchprocessed by using customized macros generated in Image-Pro Plus 5.0 (Media Cybernetics, Silver Spring, MD). Briefly, a region of interest was traced around grafted tissue in each image, and each image was then cropped to its region of interest, converted to grayscale, and processed by using a large spectral filter to enhance the appearance of vasculature while omitting presence of larger vessels (determined by filter width). For skeletal density and vessel leakage measurements images were skeletonized by using morphological filters (pixels representing branch points were excluded to divide vasculature into distinct vessel segments). Lengths of skeletal segments larger than five pixels (delineating vessels) were summed and divided by total graft area for skeletal density. Conversely, skeletal segments smaller than five pixels were summed for vessel leakage measurement.

Rat Corneal Micropocket Assay. Hydron/sucralfate pellets containing unmodified or CEP-HSA or CEP-dipeptide with or without neutralizing antibodies [1.5 $\mu \mathrm{g}$ of monoclonal mouse anti-human VEGF (R \& D Systems, Minneapolis, MN) or $4 \mu \mathrm{g}$ of monoclonal anti-CEP antibody (17)] were inserted into corneal micropockets (1 $\mathrm{mm}$ from the limbus) of Sprague-Dawley rats. Control mouse IgM $(4 \mu \mathrm{g})$ (eBioscience, San Diego, CA) and mouse IgG $(1.5 \mu \mathrm{g})$ (Southern Biotechnology Associates, Birmingham, AL) antibodies were used in control pellets for comparison with anti-CEP and anti-VEGF antibodies, respectively. Corneas were examined daily with the aid of a surgical microscope to monitor angiogenic responses to CEP-modified peptide or proteins. To photograph the angiogenic response, animals were perfused with india ink to label the vessels, and after enucleation and fixation the corneas were excised, flattened, and photographed. A positive neovascularization response was recorded only if sustained directional in-growth of capillary sprouts and hairpin loops toward the pellet was observed. A negative response was recorded when either no growth was observed or only an occasional sprout or hairpin loop showing no evidence of sustained growth was detected. All responses were compared with a negative control (pellet and pellet-containing buffer) and positive control of VEGF. For neutralization studies, responses were compared with a negative control of nonspecific mouse Ig. Angiogenic response was analyzed for peak vascular extension and total skeletal (vascular) length by using Image-Pro Plus 5.0. Before performing vessel measurements images were processed by using best-fit equalization filters, spectral filters, and large pixel-width background removal filters to enhance vasculature and eliminate image artifacts. For total skeletal length measurements, processed images were skeletonized, summing pixel lengths of resultant skeletal segments. To determine peak vessel extension, 
processed images were thresholded for vasculature, filling in holes between adjacent vessels by using morphological filters. The resulting image, a single segmented object representing the overall dimensions of the vascular bed, was analyzed for maximum boxwidth, i.e., extent of vessel penetration.

Laser-Induced CNV Assay. Laser photocoagulation-induced rupture of Bruch's membrane was used to generate $\mathrm{CNV}$ as previously described (38). Briefly, 4- to 5-week-old C57BL/6J mice were anesthetized with ketamine hydrochloride $(100 \mathrm{mg} / \mathrm{kg}$ of body weight) and xylazine (10 mg/kg of body weight) followed by $1 \%$ Tropicamide (Alcon Laboratories, Fort Worth, TX) for pupillary dilation. Three burns of 532-nm diode laser photocoagulation (50- $\mu \mathrm{m}$ spot size, 0.1-s duration, $200 \mathrm{~mW}$ ) (Oculight, Iridex, Mountain View, CA) were delivered to each retina by using a slit lamp delivery system and a handheld coverslip as a contact lens. Burns were performed in the 9,12, and 3 o'clock positions of the posterior pole of the retina. Production of a bubble at the time of lasering indicated a successful burn. Experiments were performed to investigate the effect of subretinal injections of PBS $(1 \mu \mathrm{l})$, MSA $(1 \mu \mathrm{g})$, CEP-MSA $(1 \mu \mathrm{g})$, or VEGF (20 ng) on laser-induced CNV. Animals were injected immediately after laser injury in the upper half of the retina between the burns (bubbles). Subretinal injections were also performed on mice not subjected to laser injury as a control. Two weeks later, mice were anesthetized and perfused with fluorescein-labeled dextran $\left(2 \times 10^{6}\right.$ average molecular weight $)$ (Sigma-Aldrich, St. Louis, MO), choroidal flatmounts were prepared, and CNV area was measured as described previously (38). Five mice were used for each group with three burns in each eye ( $n=15-18$ successful burns in each group). For quantitative analysis of lesion intensity and size, CNV images were batchprocessed by using a custom macro generated in Image-Pro Plus 5.1. For each image, a region of interest was traced around the lesion by using a wand tool (a manual trace was performed in a few cases where the lesion was not significantly brighter than the back-

1. Macular Photocoagulation Study Group (1991)Arch. Ophthalmol. 109, 1242-1257.

2. Macular Photocoagulation Study Group (1991)Arch. Ophthalmol. 109, 1232-1241.

3. Campochiaro, P. A. (2000) J. Cell. Physiol. 184, 301-310.

4. Beatty, S., Koh, H., Phil, M., Henson, D. \& Boulton, M. (2000) Surv. Ophthalmol. 45, 115-134.

5. Winkler, B. S., Boulton, M. E., Gottsch, J. D. \& Sternberg, P. (1999) Mol. Vis. 5, 32

6. Seddon, J. M., Willett, W. C., Speizer, F. E. \& Hankinson, S. E. (1996) J. Am. Med. Assoc. 276, 1141-1146.

7. Age-Related Eye Disease Study Research Group (2001) Arch. Ophthalmol. 119, 1417-1436.

8. Edwards, A. O., Ritter, R., III, Abel, K. J., Manning, A., Panhuysen, C. \& Farrer, L. A. (2005) Science 308, 421-424.

9. Gold, B., Merriam, J. E., Zernant, J., Hancox, L. S., Taiber, A. J., Gehrs, K., Cramer K., Neel, J., Bergeron, J., Barile, G. R., et al. (2006) Nat. Genet. 38, 458-462.

10. Hageman, G. S., Anderson, D. H., Johnson, L. V., Hancox, L. S., Taiber, A. J., Hardisty, L. I., Hageman, J. L., Stockman, H. A., Borchardt, J. D., Gehrs, K. M., et al. (2005) Proc. Natl. Acad. Sci. USA 102, 7227-7232.

11. Haines, J. L., Hauser, M. A., Schmidt, S., Scott, W. K., Olson, L. M., Gallins, P., Spencer, K. L., Kwan, S. Y., Noureddine, M., Gilbert, J. R., et al. (2005) Science 308, 419-421.

12. Klein, R. J., Zeiss, C., Chew, E. Y., Tsai, J. Y., Sackler, R. S., Haynes, C., Henning, A. K., SanGiovanni, J. P., Mane, S. M., Mayne, S. T., et al. (2005) Science 308, 385-389.

13. Bok, D. (2005) Proc. Natl. Acad. Sci. USA 102, 7053-7054.

14. Bora, P. S., Sohn, J. H., Cruz, J. M., Jha, P., Nishihori, H., Wang, Y., Kaliappan, S., Kaplan, H. J. \& Bora, N. S. (2005) J. Immunol. 174, 491-497.

15. Malek, G., Johnson, L. V., Mace, B. E., Saloupis, P., Schmechel, D. E., Rickman, D. W., Toth, C. A., Sullivan, P. M. \& Bowes Rickman, C. (2005) Proc. Natl. Acad. Sci. USA 102, 11900-11905.

16. Crabb, J. W., Miyagi, M., Gu, X., Shadrach, K., West, K. A., Sakaguchi, H., Kamei, M., Hasan, A., Yan, L., Rayborn, M. E., et al. (2002) Proc. Natl. Acad. Sci. USA 99, 14682-14687.

17. Gu, X., Meer, S. G., Miyagi, M., Rayborn, M., Hollyfield, J. G., Crabb, J. W. \& Salomon, R. G. (2003) J. Biol. Chem. 278, 42027-42035.

18. Okamoto, T., Tanaka, S., Stan, A. C., Koike, T., Kase, M., Makita, Z., Sawa, H. \& Nagashima, K. (2002) Microvasc. Res. 63, 186-195. ground). Mean intensity (range between 10 and 255 gray levels), perimeter, area, and mean diameter (pixels) were calculated for each region of interest and exported to Excel (Microsoft, Redmond, WA). Analyses were performed by a blind observer to eliminate user bias.

Cell Culture Conditions and VEGF Secretion Assay. Human ARPE19 cells were cultured in DMEM/F-12 medium, and human primary RPE cells from three different donors of ages 9, 55, and 82 years (kind gift from Janice Burke, Medical College of Wisconsin, Milwaukee, WI) were cultured in MEM, with Earle's salts and L-glutamine supplemented with $10 \%$ FBS, 100 units/ml penicillin, and $100 \mu \mathrm{g} / \mathrm{ml}$ streptomycin. Confluent cultures in 24-well plates were starved for 1 day with serum-free medium before incubating with CEP-dipeptide (0.1-10 $\mu \mathrm{M})$ and unmodified dipeptide (as controls). The CEP-dipeptide and controls were quantified by amino acid analysis (36). Supernatant medium was collected to measure VEGF secretion by using an ELISA according to the manufacturer's protocol (R \& D Systems). Concanavalin $(50 \mu \mathrm{g} /$ $\mathrm{ml}$ ) was used as a positive control for VEGF stimulation.

Statistical Analysis. Data are presented as mean \pm SD. The statistical significance of differential findings observed between experimental and control groups was determined by using one-way ANOVA or Student's $t$ test and considered to be significant if $P$ values were $<0.06$.

We are grateful to Dr. Janice Burke and Christine Skumatz (Medical College of Wisconsin, Milwaukee, WI) for the kind gift of primary human RPE cells. This work was supported in part by National Institutes of Health Grants EY016490 (to B.A.-A.), CA106415 (to B.A.-A.), EY015638 (to B.A.-A. and J.W.C.), EY06603 (to J.W.C.), EY014239 (to J.W.C.), and GM21249 (to R.G.S.); a Foundation Fighting Blindness Center grant (to B.A.-A. and J.W.C.); an American Health Association grant-in-aid (to B.A.-A.); and Biomedical Research and Technology Transfer Grant 05-29 (to B.A.-A., R.G.S., and J.W.C.).

19. Hirata, C., Nakano, K., Nakamura, N., Kitagawa, Y., Shigeta, H., Hasegawa, G., Ogata, M., Ikeda, T., Sawa, H., Nakamura, K., et al. (1997) Biochem. Biophys. Res. Commun. 236, 712-715.

20. Hoffmann, S., Masood, R., Zhang, Y., He, S., Ryan, S. J., Gill, P. \& Hinton, D. R. (2000) Invest. Ophthalmol. Vis. Sci. 41, 2389-2393.

21. Kaur, K., Salomon, R. G., O’Neil, J. \& Hoff, H. F. (1997) Chem. Res. Toxicol. 10, 1387-1396.

22. Podrez, E. A., Poliakov, E., Shen, Z., Zhang, R., Deng, Y., Sun, M., Finton, P. J., Shan, L., Febbraio, M., Hajjar, D. P., et al. (2002) J. Biol. Chem. 277, 38517-38523.

23. Podrez, E. A., Poliakov, E., Shen, Z., Zhang, R., Deng, Y., Sun, M., Finton, P. J., Shan, L., Gugiu, B., Fox, P. L., et al. (2002) J. Biol. Chem. 277, 38503-38516.

24. Subbanagounder, G., Deng, Y., Borromeo, C., Dooley, A. N., Berliner, J. A. \& Salomon, R. G. (2002) Vasc. Pharmacol. 38, 201-209.

25. Sun, M., Deng, Y., Batyreva, E., Sha, W. \& Salomon, R. G. (2002) J. Org. Chem 67, 3575-3584.

26. Gu, X., Sun, M., Gugiu, B., Hazen, S., Crabb, J. W. \& Salomon, R. G. (2003) J. Org. Chem. 68, 3749-3761.

27. Fliesler, S. J. \& Anderson, R. E. (1983) Prog. Lipid. Res. 22, 79-131.

28. Handa, J. T., Verzijl, N., Matsunaga, H., Aotaki-Keen, A., Lutty, G. A., te Koppele, J. M., Miyata, T. \& Hjelmeland, L. M. (1999) Invest. Ophthalmol. Vis. Sci. 40, 775-779.

29. Wu, J. T. (1993) J. Clin. Lab. Anal. 7, 252-255.

30. Ishibashi, T., Murata, T., Hangai, M., Nagai, R., Horiuchi, S., Lopez, P. F., Hinton, D. R. \& Ryan, S. J. (1998) Arch. Ophthalmol. 116, 1629-1632.

31. Hoffmann, S., Friedrichs, U., Eichler, W., Rosenthal, A. \& Wiedemann, P. (2002) Graefes. Arch. Clin. Exp. Ophthalmol. 240, 996-1002.

32. Anonymous (1991) Ophthalmology 98, 757-765.

33. Gragoudas, E. S., Adamis, A. P., Cunningham, E. T., Jr., Feinsod, M. \& Guyer, D. R. (2004) N. Engl. J. Med. 351:2805-2816.

34. Ng, E. W. \& Adamis, A. P. (2005) Can. J. Ophthalmol. 40, 352-368.

35. Rosenfeld, P. J., Schwartz, S. D., Blumenkranz, M. S., Miller, J. W., Haller, J. A., Reimann, J. D., Greene, W. L. \& Shams, N. (2005) Ophthalmology 112, 1048-1053.

36. Crabb, J. W., West, K. A., Dodson, W. S. \& Hulmes, J. D. (1997) Amino Acid Analysis (Wiley, Hoboken, NJ).

37. Nguyen, M., Shing, Y. \& Folkman, J. (1994) Microvasc. Res. 47, 31-40.

38. Tobe, T., Ortega, S., Luna, J. D., Ozaki, H., Okamoto, N., Derevjanik, N. L., Vinores, S. A., Basilico, C. \& Campochiaro, P. A. (1998) Am. J. Pathol. 153, 1641-1646. 